\title{
Microsporum audouinii
}

National Cancer Institute

\section{Source}

National Cancer Institute. Microsporum audouinii. NCI Thesaurus. Code C127732.

A species of anthropophilic fungi in the phylum Ascomycota that is characterized by spindle shaped macroconidia, clavate microconidia, and pitted or spiny external walls. This species is a causative agent of tinea capitis and tinea corporis. 